\title{
AN EXTENSION OF LINDELÖF'S THEOREM TO MEROMORPHIC FUNCTIONS
}

\author{
S. M. SHAH ${ }^{1)}$
}

(Received January 6, 1960)

1. Introduction. If $f(z)$ be an entire function of finite order $\rho$, then Lindelöf has obtained a set of conditions in order that $f(z)$ may be of maximum, mean or minimum type $[2 ; 1,25-30]$. A theorem of the similar nature for meromorphic functions is stated by Valiron [8] and Hari Shanker has recently [3] extended the results of Lindelöf by taking comparison function $r^{\rho} L(r)$. In this note we prove three theorems which will include the theorems of Lindelöf, Valiron and Hari Shanker as special cases.

Let $f(z)$ be a meromorphic function of finite order $\rho$. We have

$$
f(z)=z^{k} \exp \left(C z^{p_{3}}+\cdots\right) \prod_{1}^{\infty} E\left(z / a_{n}, p_{1}\right) / \prod_{1}^{\infty} E\left(z / b_{n}, p_{2}\right),
$$

where $Q(z)=C z^{p_{3}}+\cdots \cdots$ is a polynomial of degree $p_{3} \leqq[\rho]$. Write

$$
n(r)=n(r, 1 / f)+n(r, f), N(r)=N(r, 1 / f)+N(r, f) .
$$

When $\rho>0$, and $N(r)$ is of order $\rho$, we define a proximate order $\rho(r)$ for $N(r)$ as follows.

(i) $\rho(r)$ is differentiable for $r>r_{0}$, except at isolated points at which $\rho^{\prime}(r-0)$ and $\rho^{\prime}(r+0)$ exist.

$$
\begin{aligned}
& \lim _{r \rightarrow \infty} \rho(r)=\rho . \\
& \lim _{r \rightarrow \infty} r \rho^{\prime}(r) \log r=0 . \\
& \lim _{r \rightarrow \infty} \sup N(r) / r^{\rho(r)}=1 .
\end{aligned}
$$

For the existence of a proximate order see [6] where $\rho(r)$ is constructed with $\log M^{\prime}(r)$; the argument given there can be utilised to construct $\rho(r)$ with the above properties (i) - (iv).

When $\rho$ is integer, we can write $f(z)$ in the form

$$
\begin{aligned}
f(z) & =z^{\kappa} \exp \left(c z^{\rho}+\cdots\right) \cdot \prod_{1}^{\infty} E\left(z / a_{n}, \rho\right) / \prod_{1}^{\infty} E\left(z / b_{n}, \rho\right) \\
& =z^{k} \exp \left(c z^{\rho}+\cdots\right) P_{1} / P_{2} \text { (say) }
\end{aligned}
$$

1) Abstract presented to Indian Math.Soc., Dec. 1959. 
where if $f$ has no poles, $P_{2}$ is to be replaced by 1 , and if $f$ has a finite number of poles then $\quad P_{2}=\prod_{1}^{M} E\left(z / b_{n}, \rho\right)$.

Similarly when $\quad \lim _{r \rightarrow \infty} n(r, 1 / f)<\infty$.

THEOREM 1. If $\rho$ is integer and $N(r)$ is of order $\rho$, then

$$
T(r, f)=\frac{r^{\rho}}{\rho \pi}\left|c \rho+\sum_{\left|a_{n}\right|<r} a_{n}^{-\rho}-\sum_{\left|b_{n}\right|<r} b_{n}^{-\rho}\right|+O\left(r^{\rho(r)}\right)
$$

where $f(z)$ is given by (1.2). If $N(r)$ is of order less than $\rho$, then we have from (1.1)

$$
p_{3}=\rho, C \neq 0, T(r, f)=\frac{r^{\rho}}{\pi}|C|+O\left(r^{\mu}\right), \mu<\rho .
$$

THEOREM 2. Let $f(z)$ be a meromorphic function of integer order $\rho$ and let $L(r)$ be a slowly changing positive function [4; pp. 52-54] and write

(1.5) $\limsup _{r \rightarrow \infty} S(r) / r^{\rho} L(r)=S_{L}$;

where

$$
S(r)=\frac{r^{\rho}}{\rho \pi}\left|c \rho+\sum_{\left|a_{n}\right|<r} a_{n}^{-\rho}-\sum_{\left|b_{n}\right|<r} b_{n}^{-\rho}\right| .
$$

(i) If $N(r)$ is of order $\rho$, then

$$
\begin{aligned}
& 0<T_{L}<\infty \Longleftrightarrow 0<\max \left(n_{L}, S_{L}\right)<\infty, \\
& T_{L}=\infty \Longleftrightarrow \max \left(n_{L}, S_{L}\right)=\infty, \\
& T_{L}=0 \Longleftrightarrow \max \left(n_{L}, S_{L}\right)=0 .
\end{aligned}
$$

(ii) If $N(r)$ is of order less than $\rho$, then

$$
T(r) \sim S(r) \sim r^{\rho}|C| / \pi ; n(r)=O\left(r^{\alpha}\right), \alpha<\rho .
$$

THEOREM 3. Let $f(z)$ be a meromorphic function of order $\rho$.

(i) If $\rho>0$ be non-integer, and $T_{L}$ etc. be defined as in (1.5), then

$$
\begin{aligned}
& 0<T_{L}<\infty \Longleftrightarrow 0<n_{L}<\infty, \\
& T_{L}=\infty \Longleftrightarrow n_{L}=\infty, \\
& T_{L}=0 \Longleftrightarrow n_{L}=0 .
\end{aligned}
$$

(ii) When $\rho=0$, let the comparison function be $L(r)=(\log r) L_{1}(r)$ where $L_{1}(r)$ is slowly changing and $\uparrow \infty$ with $r$. Write 


$$
T_{L}=\limsup _{r \rightarrow \infty} T(r) / L(r) ; N_{L}=\limsup _{r \rightarrow \infty} \frac{\{N(r, 1 / f)+N(r, f)\}}{L(r)} .
$$

Then

$$
T_{L} \leqq N_{L} \leqq 2 T_{L},
$$

and the relations (1.9) hold with $N_{L}$ instead of $n_{L}$. If $f(z)$ be entire func. tion of zero order, then $T_{L}=N_{L}$.

2. Proof of Theorem 1. Write (1.2) in the form

$$
f(z)=\phi_{0} \phi_{1} \phi_{2} \phi_{3} \phi_{4}^{-1} \phi_{5}
$$

where

$$
\begin{gathered}
\phi_{0}(z)=z^{\kappa} \exp \left(c_{1} z^{\rho-1}+\cdots\right), \\
\phi_{1}(z)=\exp \left\{c z^{\rho}+\frac{z^{\rho}}{\rho}\left(\sum_{\left|a_{n}\right|<2 r} a_{n}^{-\rho}-\sum_{\left|b_{n}\right|<2 r} b_{n}^{-\rho}\right)\right\}, \\
\phi_{2}(z)=\prod_{1}^{n_{0}} E\left(z / a_{n}, \rho-1\right) / \prod_{1}^{n_{0}} E\left(z / b_{n}, \rho-1\right), \\
\phi_{3}(z)=\prod_{n>n_{0}}^{\left|a_{n}\right|<2 r} E\left(z / a_{n}, \rho-1\right), \\
\phi_{4}(z)=\prod_{n>n_{0}}^{\left|b_{n}\right|<2 r} E\left(z / b_{n}, \rho-1\right), \\
\phi_{5}(z)=\prod_{\left|a_{n}\right| \geq 2 r} E\left(z / a_{n}, \rho\right) / \prod_{\left|b_{n}\right| \geq 2 r} E\left(z / b_{n}, \rho\right) .
\end{gathered}
$$

Now for $\phi_{0}, \phi_{2}$

$$
T(r)=O\left(r^{\rho-1}\right)+O(\log r) .
$$

Further

$$
\log ^{+}\left|\phi_{3}(z)\right|=O\left(N(r, 1 / f)+r^{\rho-1} \int_{r_{0}}^{2 r} n(x, 1 / f) x^{-\rho} d x+n(2 r, 1 / f)\right) .
$$

Hence

$$
T\left(r, \phi_{3}\right)=O\left(r^{\rho(r)}\right) .
$$

Similarly

$$
\begin{gathered}
T\left(r, \phi_{4}\right)=O\left(r^{\rho(r)}\right), \\
T\left(r, \phi_{5}\right)=O\left(r^{\rho+1} \int_{2 r}^{\infty} x^{\rho(x)-\rho-2} d x\right)=O\left(r^{\rho(r)}\right) .
\end{gathered}
$$


Hence

$$
\begin{aligned}
T(r, f) & =\frac{r^{\rho}}{\rho \pi}\left|c \rho+\sum_{\left|a_{n}\right| \leqq r} a_{n}^{-\rho}-\sum_{\left|b_{n}\right| \leqq r} b_{n}^{-\rho}\right|+O\left(r^{\rho(r)}\right) \\
& =S(r)+O\left(r^{\rho(r)}\right) .
\end{aligned}
$$

If $N(r)=O\left(r^{\alpha}\right)$, where $\alpha<\rho$ then

$$
T\left(r, P_{i}\right)=O\left(r^{\beta}\right), i=1,2, \alpha<\beta<\rho .
$$

Hence

$$
P_{3}=\rho, C \neq 0, T(r, f)=\frac{|C| r^{\rho}}{\pi}+O\left(r^{\mu}\right), \mu<\rho .
$$

3. Proof of Theorem 2. (i) Suppose $N(r)$ is of order $\rho$. If $n_{L}<\infty$, then we obtain as in the first part of Theorem 1 ,

$$
T(r, f)=S(r)+O\left(r^{\rho} L(r)\right)
$$

and if $n_{L}=0$, then

$$
T(r, f)=S(r)+o\left(r^{\circ} L(r)\right) .
$$

Hence if $S_{L}<\infty$, then $T_{L}<\infty$. If $T_{L}<\infty$, then $T(r)<A r^{\rho} L(r), N(r)$ $<A_{1} r^{\rho} L(r), n(r)<A_{2} r^{\rho} L(r)$. Hence $n_{L}<\infty$ and from (3. 1) $S_{L}<\infty$. If $n_{L}>0$ then $T_{L}>0$. If $T_{L}>0$, then $\max \left(n_{L}, S_{L}\right)>0$, for if $n_{L}=0$, then from (3. 2), $T_{L}=S_{L}>0$. If $T_{L}=\infty$, then $\max \left(n_{L}, S_{L}\right)=\infty$, for if this expression is less than $\infty$, then from (3.1) we get $T_{L}<\infty$. If $n_{L}=\infty$ then $T_{L}=\infty$ and if $S_{L}=\infty, n_{L}<\infty$ then from (3.1) $T_{L}=\infty$. If $T_{L}=0$, then $n_{L}=0$ and from (3.2) $S_{L}=0$. If $n_{L}=S_{L}=0$ then $T_{L}=0$.

(ii) Since $N(r)$ is of order $<\rho, \sum a_{n}^{-p}, \sum b_{n}^{-p}$ are both convergent, and if $f$ has an infinity of zeros and an infinity of poles,

$$
c \rho=C \rho-\sum_{1}^{\infty} a_{n}^{-\rho}+\sum_{1}^{\infty} b_{n}^{-\rho} .
$$

Hence

$$
S(r)=\frac{r^{\rho}}{\rho \pi}\left|C \rho-\sum_{\left|a_{n}\right|>r} a_{n}^{-\rho}+\sum_{\left|b_{n}\right|>r} b_{n}^{-\rho}\right|
$$

and so

$$
S(r) \sim r^{\rho}|C| / \pi
$$

Similarly we can prove (3.3) when $f$ has a finite number of poles or zeros 
or both. Hence from (1.4) and (3.3)

$$
T(r) \sim S(r) ; n(r)=O(N(2 r))=O\left(r^{\alpha}\right), \alpha<\rho .
$$

4. Proof of Theorem 3. (i) We have from (1. 1), [7]

$$
\begin{aligned}
T(r, f) & \leqq O\left(r^{p_{3}}\right)+O(\log r)+\log M\left(r, P_{1}\right)+\log M\left(r, P_{2}\right) \\
& \leqq O\left(r^{\mu}\right)+O(\log r)+A \int_{0}^{\infty} \frac{n(x) r^{1+\mu}}{x^{1+\mu}(x+r)} d x
\end{aligned}
$$

where $\mu=[\rho]$. Hence if $n_{L}<\infty$,

$$
\begin{aligned}
& T(r, f) \leqq A_{1}\left\{r^{\mu}\right.+\log r+r^{\mu} \int_{1}^{r} x^{\rho-\mu-1} L(x) d x \\
&\left.+r^{1+\mu} \int_{r}^{\infty} x^{\rho-\mu-2} L(x) d x\right\} \\
& \leqq A_{1}\left\{r^{\mu}+\log r+\frac{r^{\rho} L(r)}{\rho-\mu}+\frac{r^{\circ} L(r)}{1+\mu-\rho}\right\} \\
&<A_{2} r^{\rho} L(r),
\end{aligned}
$$

and (1.9) follows provided $n_{L}<\infty$. If $n_{L}=\infty$ then $T_{L}=\infty$ and from above if $T_{L}=\infty$ then $n_{L}=\infty$.

(ii) We have [5]

$$
\begin{gathered}
N(r) \leqq 2 T(r, f)+O(1), \\
T(r, f) \leqq\{1+o(1)\} r \int_{r}^{\infty}\left\{N(t) / t^{2}\right\} d t
\end{gathered}
$$

and (1.10) follows. If $f$ be entire then $N(r) \leqq T(r, f)+O(1), T_{L}=N_{L}$.

5. Remarks. (i) If $f$ is entire and $p_{1}=\rho$ then from (1.3) we have, since $c=C$,

$$
T(r, f)=\frac{r^{\rho}}{\rho_{\pi}}\left|C \rho+\sum_{\left|a_{n}\right|<r} a_{n}^{-\rho}\right|+O\left(r^{\rho(r)}\right)
$$

Further since

$$
T(r, f) \sim T(r, f P)
$$

where $P$ is any polynomial, (4.1) holds also for functions with a finite number of poles and $p_{1}=\rho$. We can get this result directly from (1.3) for we will have

$$
T(r, f)=\frac{r^{\rho}}{\rho_{\pi}}\left|\left(C+\frac{1}{\rho} \sum_{1}^{k} b_{n}^{-\rho}\right) \rho+\sum_{\left|a_{n}\right|<r} a_{n}^{-\rho}-\sum_{1}^{k} b_{n}^{-\rho}\right|+O\left(r^{\rho(r)}\right) .
$$


A similar remark applies when $f$ has a finite number of zeros.

(ii) The formula (1.3) is useful when $S(r)$ is large compared to $r^{\rho(r)}$. For instance if

$$
f(z)=\Gamma(z), S(r) \sim\{r \log r\} / \pi, r^{\rho(r)}=O(r) .
$$

But for functions of the form (1.2) with

$$
c \rho+\sum_{\left|a_{n}\right|<r} a_{i \imath}^{-\rho}-\sum_{\left|b_{n}\right|<r} b_{n}^{-\rho}=O(1) ; N(r)>\alpha r^{\circ}, \alpha>0, r>r_{0},
$$

(1.3) does not give much information.

(iii) If $f$ be meromorhic function of integer order $\rho$ and such that $\max \left(p_{1}, p_{2}\right)=\rho-1$, then we get from (1.3)

$$
T(r, f)=\frac{r^{\rho}}{\rho_{\pi}}\left|C \rho-\sum_{\left|a_{n}\right|>r} a_{n}^{-\rho}+\sum_{\left|b_{n}\right|>r} b_{n}^{-\rho}\right|+O\left(r^{\rho(r)}\right) .
$$

If $f$ be entire function with $p_{1}=\rho-1, p_{3}<\rho$ and such that $n_{L}<\infty$, then from (3.1) and (4.2)

$$
T(r, f)=\frac{r^{\rho}}{\rho_{\pi}}\left|\sum_{\left|a_{n}\right|>r} a_{n}^{-\rho}\right|+O\left(r^{\rho} L(r)\right) .
$$

It is not necessary to suppose here that $L(r)$ be monotone except when $\rho$ $=0$ (see Theorem 3(ii)).

\section{REFERENCES}

[1] R.P. BOAS, Entire Functions, Academic Press, New York, 1954.

[2] E.F.LINDELÖF, Sur les fonctions entieres d'ordre entier, Ann. Sci. Ecole Norm. Sup. (3), 22(1905), 365-395.

[3] HARI SHANKER, On Lindelöf's theorem on entire functions, Jour. Ind. Math. Soc., 27(1958), 137-147.

[4] G. H. HARDY AND W. W. Rogosinki, Note on Fourier seris, Quart. J. of Maths. (Oxford Series), 16(1945), 49-58.

[5] S. M. SHAH, A note on meromorphic functions, Math. Student, 12(1944), 67-70.

[6] S. M. SHAH, On proximate orders of entire functions, Bull. Amer. Math Soc., 52(1946), 326-328.

[7] S. M. SHAH, Some theorems on meromorphic functions, Proc. Amer. Math. Soc., 1(1951), 694-698.

[8] G. VALIRON, Remarques sur les valuers exceptionnelles des fonctions méromorphes, Rend. Circ. Mat. Palermo, 57(1933), 71-86.

NORTHWEstern UNIVERSITY, EVANSTON, U.S. A.

$$
\text { AND }
$$

Muslim UNIVERSITY, ALIGARH, INDIA. 